TURIZAM

Volume 21, Issue 1

45-64 (2017)

ORIGINAL

SCIENTIFIC PAPER

\title{
Impact of Organizational Conflict on Employee Job Performance in Selected Hotels (in Lagos Nigeria)
}

\author{
Nwokorie Edwin Chigozie ${ }^{A}$ \\ Received: February 2017 | Accepted: April 2017 \\ DOI: 10.18421/TRZ21.01-04
}

\begin{abstract}
Three first class establishments in Lagos Nigeria were investigated with a study population of 122 from which a sample size of 110 was drawn to model the impact of organizational conflict on employee job performance in the hotel industry. A 61 point questionnaire was prepared, using a four-point Likert rating scale for data collection. Data was analyzed using inferential statistics and hypotheses were tested in Chi-square statistics. Results showed that unclear responsibilities, poor reward system, and lack of group cohesiveness, are responsible for organizational conflicts; with the consequential impact on employee job commitment, service quality, customer satisfaction and organizational performance, whereas outcomes generate influence on employee job satisfaction and job performance, thus creating turnover intentions and further generating organizational conflict. The research suggests a strengthened supportive mechanism from the human resource department and forestalling distrust among working groups, which will reduce the impact of work stressors on psychological states.
\end{abstract}

Keywords: customer satisfaction, employee performance, employee turnover, hotel industry, organizational conflict.

\section{Introduction}

Extensive studies in human resource management have expressly addressed the issue of organizational conflict and its negative impact on various facets of the hospitality industry; as the excessive level of organizational conflict has become one of the primary concerns in the lodging and food service industry $(\mathrm{Ku}, 2007)$. The most prominent effects are the quality of the products and services, excessive expenses incurred as a result of recruitment and replacement resulting from employee turnover, and most importantly, the loss of profit due to poor overall organizational performance (Berta, 2004). Ku (2007) equally stated that the hospitality industry is experiencing an all-time high turnover rate compared with other industries, and is doing a less favourable job in managing turnover.

Since the characteristics of each industry are different from others and the reasons employees leave that specific industry are complex, some factors leading to employees' intention to

A Department of Hospitality Management and Technology, The Federal Polytechnic Ilaro, Nigeria, eddygozie@gmail.com 
leave in one industry may not necessarily apply to the others. It has been presented evidently that job-content factors are significantly related to turnover $(\mathrm{Ku}, 2007)$, and their relationships to job satisfaction are also associated with turnover. However, there is no consensus on the consequences of turnover in the hospitality industry hence, this study investigated the impact of organizational conflict on employee job performance and its implication on service quality, customer satisfaction, employee job satisfaction and employees' intention to leave; and the causes of organizational conflicts in the hospitality industry.

Given that there are very high and unacceptable rates of employee turnover in the hospitality industry, it is imperative to minimize the role of conflict as a causal effect. Although conflict does (and in some cases should) occur, it should not lead to an employee seeking to terminate his employment. Employee turnover in the hospitality industry is a major problem on a global scale (Nicolaides, 2010) and this is why organizational conflict management should be minimized.

\section{Research Objectives}

This study examined the impact of organizational conflict on employee job performance, how organizational conflicts arise in a hospitality organization, which when discovered will ultimately expose how they can be effectively forestalled by managers at various levels.

The specific objectives were to;

1. Evaluate the impact of organizational conflict on employee job performance.

2. Evaluate the impact of organizational conflict on the performance of a hospitality organization.

3. Examine how organizational conflict can impact on the behaviour of employees' job commitment which may likely influence service quality and customer satisfaction.

4. Examine the relationship between organizational conflict and employees' intention to leave the job.

\section{Research Hypotheses}

$\mathbf{H}_{\mathbf{1}}$ : There are no significant impact of organizational conflict on employee job performance in the hospitality industry.

$\mathbf{H}_{\mathbf{2}}$ : There are no significant impact of organizational conflict on the overall performance of the hospitality organization.

$\mathbf{H}_{3}$ : Behaviours of employee poor commitment have no negative effect on service quality and customer satisfaction in the hospitality industry.

$\mathbf{H}_{4}$ : There is no significant relationship between organizational conflicts and employees' intention to leave the job.

\section{Review of Related Literature}

\section{Empirical Literature}

The relationship between organizational conflicts and employee turnover and the consequence has been well document and reveals, for instance, that high employee turnover hurt both organizations and their remaining employees in terms of work performance and job disruption (Scott, et al., 1999; Ku, 2007). Moreover, considerable research has been devoted to 
addressing the issue related to organizational conflict and employee turnover both empirically and theoretically. For instance, studies have examined the impact and causes of turnover (Birdir, 2002; Hinkin, Tracey, 2000; Simons, Hinkin, 2001), and numerous predictive and turnover path models and their relationships with various antecedents (Maertz, Griffeth, 2004; Tett, Mayer, 1993).

a) Role Conflict: According to Stryker and Macke (2008), the term "role conflict" has been applied to different conceptual process aspects, such as "(1) competing demands arising from different parts of a given role set, (2) conflicting reactions of the same individuals to the same types of behaviours, (3) differences in the expectations of others and (4) differences between role expectations and individuals' self-concepts". This study focuses only on that aspect of role conflict defined by temporal convergences of competing demands arising from different roles; for example, work and family. In other words, role conflict occurs when demands associated with one role interfere directly with one's ability to satisfy the demand of another role.

Empirical studies on the consequences of role conflict have been conducted in complex organization settings exploring the effect of role conflict on psychological health (Behrman, Perreault, 2004; Boles, Babin, 2006; Dubinsky, Hartley, 2006), work-related attitudes and behaviour (Bedeian, Amenakis, 2001; Good, et al, 1998). Furthermore, most turnover models have postulated that, role conflict, role ambiguity, and role overload will ultimately relate to the explanation of turnover directly or indirectly (Bedeian, Amenakis, 2001).

In different analytical framework, the relationship between role conflict and job related attitudes and behaviours is inconsistent. Bedeian and Armenakis (2001) and Netemeyer, Johnston, and Burton (200o) reported weak causal paths from role conflict to propensity to leave; and Bedeian, Burke and Meffett (2008) further reported the relationship between role conflict and job satisfaction was not significant.

b) Role Ambiguity: Spector (1997) wrote that, "Role ambiguity is the degree of certainty the employee has about what his or her functions and responsibilities are". There are two sources of ambiguity as stated by Kahn, Wolfe, Quinn, Snoek, and Rosenthal (2004). Objective ambiguity is due to the lack of information needed for role definition and role performance, while subjective ambiguity is associated with the social and psychological aspects of role performance (Woods, Macaulay, 2009).

Numerous studies have indicated that role ambiguity was found to be significantly negatively related to job satisfaction (Baroudi, 2005). However, in the relationship between role ambiguity and intention to leave, different results were found that role ambiguity was the most dysfunctional variable in turnover intention (Bedeian, Armenakis, 2001; Netemeyer, et al., 200o), while a couple of studies stated that there was a weak causal relationship between role ambiguity and propensity to leave (Bedeian, Armenakis, 2001; Behrman, Perreault, 2004). In order to further enhance the understanding of the consequences of role conflict and ambiguity for work related attitudes and outcomes, studies also suggested that there are additional factors, such as different work settings, differences in contexts, and tasks that need to be incorporated in future research (Hecht, 2011; Hood, Millazzo, 2013).

c) Work Schedule: Studies on the impact of shiftwork on personal/family life have demonstrated the disruptive influence on physical and mental health problems (Schulz, et al., 2007). In most organizational settings, hours worked, especially during weekends and weeknights, have been found to be significantly correlated with turnover intentions 
and provided the explanation of a large percentage of the variance in intention to leave (Almer, Kaplan, 2000; Orpen, 2001; Woods, Macaulay, 2009).

The relationship between work scheduling and the work related outcomes, such as unfavourable turnover has been one of the major concerned subjects when the human resource personnel is considering the employee retention programme (Schulz, et al., 2007). Furthermore, weekend and weeknight hours worked, along with job satisfaction, were found to have direct linkages with turnover intentions (Jamal, 2001). The same implication that shiftwork was related to withdrawal behaviour was found in the study of nurses and industrial workers (Kazerooni, 2005). The hospitality industry, which has a notorious history of long hours and low pay, has different assessments regarding the influence of working hours and shifts (Nwokorie, Ezeibe, 2016).

d) Pay Satisfaction: Pay satisfaction is assumed to be predictive of absenteeism and turnover (Cotton, Tuttle, 2006; Guthrie, 2000; Lum, et al., 2008). A number of studies have included pay satisfaction as a component of job satisfaction (Lum, et al., 2008). Allen, Drevs, and Ruhe (2009) found that one of the top three reasons employees leave their organizations is the opportunity for higher pay at another organization. Pay dissatisfaction often leads to decreased motivation, morale, and work quality (Leonard, 2001). Pay satisfaction has been identified as a determinant to job satisfaction, organizational commitment and behavioural intentions to leave the organization (Lum, et al., 2008). Pay is often used in organizations to motivate employees (Lum, et al., 2008). Employees view it as an important reward or outcome. Consequences of pay dissatisfaction include negative employee behaviours (Lum, et al., 2008). Such behaviours are turnover, absenteeism, willingness to strike, and lowered job performance. In the hotel industry, Woods, Heck and Sciarini (2008) found that rate of pay was the top reason of the most important internal causes of turnover. In addition, the major cause of the most important external causes of turnover was "better pay elsewhere" (Woods, et al., 2008).

e) Intention to Leave: Fishbein and Ajzen (1985) proposed a turnover model based on the theory of planned behaviours. Fishbein and Ajzen postulated that the belief an individual may have is related to the person's attitude, and would finally lead to a specific action. Mitchel, Holtom, Lee, Sablynski, and Ere (2001) agreed that the Fishbein and Ajzen attitude model was based on the premise that "employees' behaviours could be influenced by the extent to which other people expected them to behave in a certain manner and is further reinforced by personal motivation on the person's part to comply with such expectations".

Due to the difficulty of predicting actual turnover before it occurs, the best alternative would be to measure variables that consistently and immediately precede voluntary turnover, such as employees' intention to quit (Lambert, et al., 2001). Spencer, Steers and Mowday (2003) agreed that the Fishbein and Ajzen attitude theory might have a superior ability to predict turnover, compared to several other models. Richer, Blanchard and Vallerand (2002) whose study was based on a motivational theory also confirmed that overtime, turnover intentions translate into actual turnover behaviour.

f) Employee Job Performance and Satisfaction: The performance of an employee in a given organization can be affected by a variety of reasons including long working hours which has become a traditional norm in the hospitality industry (Nwokorie, 2016). Furthermore, working couples with children are experiencing more imbalance in work and family life and desire to reduce the working hours in the industry (Maqsood, et al., 2012). Similarly, Deutsch, Coleman and Marcus (2011) wrote that 'when the employee con- 
sistently faces work-family conflict, he is exposed to emotional exhaust and this ultimately drags the employee to turnover intentions, and job performance is also affected. The experience of negative work-to-home disturbance is linked with the less employees' capability to deal with both realms and cheaper fulfilment with work-family stability (Hecht, 2011).

g) Work Family Conflict and Intention to Leave: There is a consensus in the literature regarding the way work-family conflict considerations affect turnover intention either directly or moderating; however, few turnover models have addressed work-family conflicts as an important factor in the turnover process (Howard, et al., 2004; Linden, 2005). Regardless of applying various turnover models and different theoretical orientations, intention to quit and absenteeism have been linked to work-family conflict, and several studies have revealed a significant relationship between work-family conflict and intention to leave. Good, Page, and Young (1996) found a direct relationship between work-family conflict and intention to leave among entry-level retail managers but not the upper-level group. Good, et al. (1998) reported that although the relationship between work-family conflict and intention to leave was positive, it was quite weak. In addition, Boyar, Maertz, Pearson and Keough (2003) further examined the effect of work-family conflict and family-work conflict on turnover intentions separately and found that both were significant in predicting turnover intentions.

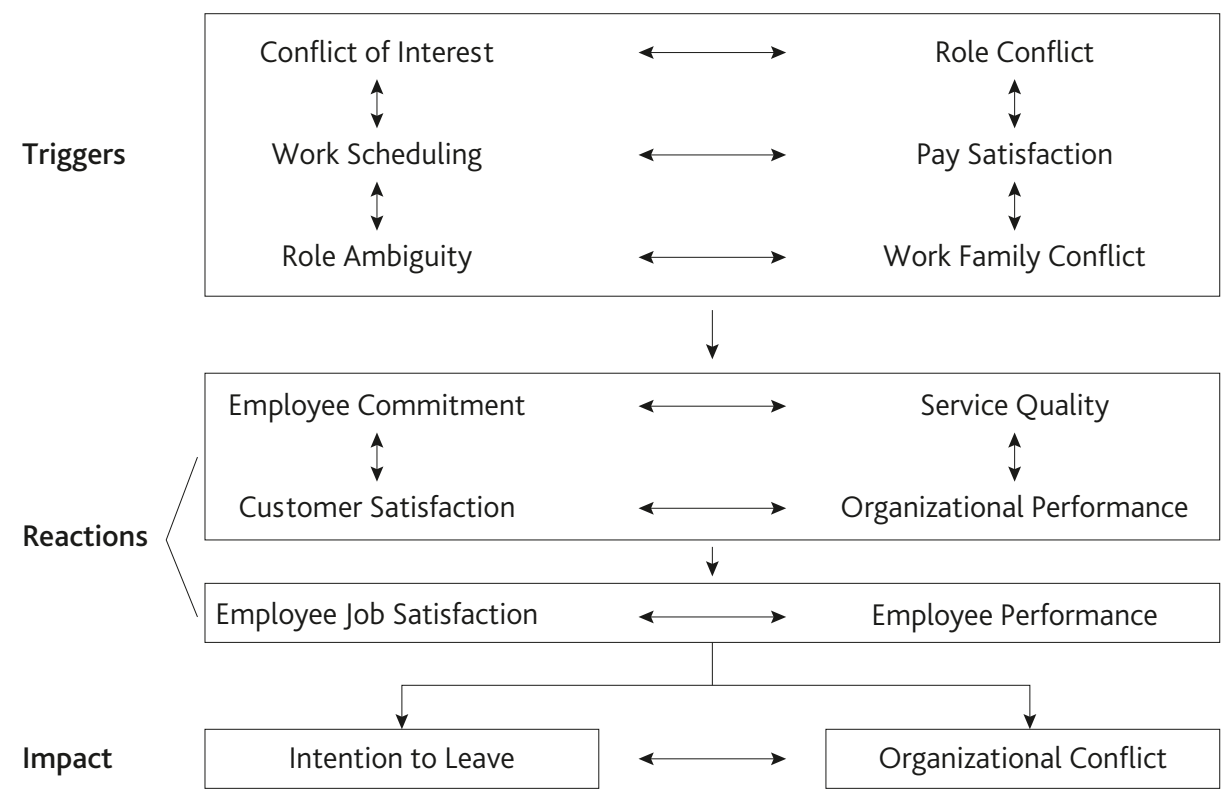

Figure 1. Research Model (Adopted from Ku, 2007)

\section{Methodology}

The research model developed for the study utilized a three-stage quantitative approach and a cross-sectional survey research design. The study employed a questionnaire (using a fourpoint Likert rating scale) which was premised upon relevant literature and survey models of past researchers to create the new instrument. To this end, the questionnaire was prepared 
in 10 sub-scales, in the three-stage approach, to address the research objectives and form the basis for testing the research hypotheses. The elements in the sub-scale were determinants of personal employee performance and turnover intentions-related decisions such as; job stressors, customer friendliness, family support, and pay expectations; and were adopted from previous research, as follows: conflict of interest and causes of conflict (Rizzo, et al., 1970), role conflict (Bedeian, Amenakis, 2001; Good, et al., 1998), work scheduling (Wood, Macaulay, 2009; Schulz, et al., 2007; Nwokorie, Ezeibe, 2016), pay satisfaction (Lum, et al., 2008), work-family conflict (Good, et al., 1998; Maertz, et al., 2003), employee job satisfaction (Nwokorie, 2016; Hecht, 2011), service quality and customer satisfaction (Nwokorie, Ezeibe, 2016), organizational performance (Ku, 2007), and intention to leave (Mitchel, et al., 2001; Lambert, et al., 2001).

\section{Population of Study}

The population for this study consists of management staff, frontline employees at various levels of the industry as well as customers of the study establishments. The employees' positions cut across various types of managerial and customer service roles. This is considered to be well represented across job classifications and can serve the research purpose regarding the characteristic of the target population's organizational role. The choice to study the establishments was premised on their perceived standard as first class hotels in the country.

\section{Sample Size}

The target population is made up of 34 frontline employees, 38 management employees, and 50 customers of the selected establishments. The determination of the sample size is based on the 122 study population and was determined using the Taro Yamen formula, which is thus stated:

$$
n=\frac{N}{1+N(e)^{2}}
$$

where:

$n=$ sample size

$N=$ finite population

$1=$ constant

$e=$ correction factor level (level of significance of error assumed to be 0.05) (Schawnms, 1994).

Table 1. Population of Frontline Employees

\begin{tabular}{|c|c|c|c|c|}
\hline Section & Hotel A & Hotel B & Hotel C & Total \\
\hline Front Office & 4 & 4 & 6 & 14 \\
\hline Food Service & 3 & 3 & 4 & 10 \\
\hline Reservation & 3 & 4 & 3 & 10 \\
\hline Grand Total & 10 & 11 & 13 & 34 \\
\hline
\end{tabular}

Source: Field survey, 2017 
Table 2. Population of Managers/Management Staff

\begin{tabular}{|c|c|c|c|c|}
\hline Section & Hotel A & Hotel B & Hotel C & Total \\
\hline Front Office & 4 & 4 & 4 & 12 \\
\hline Housekeeping & 3 & 3 & 3 & 9 \\
\hline Food Service & 3 & 3 & 3 & 9 \\
\hline Reservation & 2 & 3 & 13 & 38 \\
\hline Grand Total & 12 & 13 & & \\
\hline
\end{tabular}

Source: Field survey, 2017

Table 3. Population of Customers

\begin{tabular}{|c|c|c|c|c|}
\hline Section & Hotel A & Hotel B & Hotel C & Total \\
\hline & 16 & 19 & 15 & 50 \\
\hline Grand Total & 16 & 19 & 15 & 50 \\
\hline
\end{tabular}

Source: Field survey, 2017

\section{a) Frontline Employees}

$$
\begin{aligned}
& n=\frac{34}{1+N(0.05)^{2}} \\
& n=\frac{34}{1+(34 \cdot 0.0025)} \\
& n=\frac{34}{1+0.085} \\
& n=\frac{34}{1.085} \\
& n=31
\end{aligned}
$$

\section{b) Management/Senior Staff}

$$
\begin{aligned}
& n=\frac{38}{1+N(0.05)^{2}} \\
& n=\frac{38}{1+(38 \cdot 0.0025)} \\
& n=\frac{38}{1+0.095} \\
& n=\frac{38}{1.095} \\
& n=35
\end{aligned}
$$




\section{c) Customers}

$$
\begin{aligned}
& n=\frac{50}{1+N(0.05)^{2}} \\
& n=\frac{50}{1+(50 \cdot 0.0025)} \\
& n=\frac{50}{1+0.125} \\
& n=\frac{50}{1.125} \\
& n=44
\end{aligned}
$$

\section{Analysis of Data}

\section{Response Rate}

A total of 110 questionnaires were delivered to the respondents which resulted to $100 \%$ effective sample size. About 108 questionnaires were returned, yielding a raw response rate of $98.2 \%$. Net effective response rate was also $98.2 \%$. Unusable number of responses were (four) found among those of the customer responses, while total number of usable questionnaires were 105, representing a usable response rate of $97.2 \%$, while the net response rate was $95.5 \%$ as presented in Table 4.

Table 4. Response Rate

\begin{tabular}{|l|c|}
\hline Total number of surveys & 110 \\
\hline Number not delivered & 0 \\
\hline Percentage not delivered & $0 \%$ \\
\hline Effective sample size $^{1}$ & 110 \\
\hline Surveys returned & 108 (65 employees \& 43 customers) \\
\hline Raw response rate ${ }^{2}$ & $98.2 \%$ \\
\hline Net effective response rate $^{3}$ & $98.2 \%$ \\
\hline Number unusable & 3 (customers' response) \\
\hline Net number usable & 105 \\
\hline Usable response rate & $97.2 \%$ \\
\hline Net response rate & \\
\hline
\end{tabular}

Notes: 1: Total number of surveys minus number not delivered; 2: Surveys returned $\div$ total number of surveys; 3: Surveys returned $\div$ effective sample size; 4 : Net number usable $\div$ effective sample size; 5 : Net number usable $\div$ total number of surveys

Source: Field Survey, 2017

\section{Characteristics of Sample}

Eighteen out of the 35 members of the managers were male respondents (51.4\%) while 17 (48.6\%) of the management staff were females. The 31 frontline employees were made up of 15 (48.4\%) male respondents and 16 (51.6\%) females. Out of 44 customers, 28 (55.5\%) were males while 16 of them were females, representing $36.4 \%$ as presented in Table 5 . 
Table 5. Characteristics of Sample

\begin{tabular}{|l|c|c|c|c|c|c|}
\hline Strata of Sample & Male & \% Male & Female & \% Female & Sub-(total) & \% Total \\
\hline Managers & 18 & 51.4 & 17 & 48.6 & 35 & 100 \\
\hline Frontline employees & 15 & 48.4 & 16 & 51.6 & 31 & 100 \\
\hline Customers & 28 & 63.6 & 16 & 36.3 & 44 & 100 \\
\hline Total & 61 & 65.55 & 49 & 45.55 & 110 & 100 \\
\hline
\end{tabular}

n=110; Source: Field Survey, 2017

\section{Respondents Distribution by Establishments}

From the 105 usable responses, Hotel 'A' has nine frontline employees (8.6\% respondents), 12 managers (11.4\% respondents) and 12 customers ( $11.4 \%$ respondents). Hotel 'B' provided 10 frontline employees (9.5\% respondents), 11 managers (10.5\% respondents), and 14 customers (13.3\% respondents). Comparatively, Hotel 'C' has 12 frontline employees (11.4\% respondents), 11 managers (10.5\% respondents), and 14 customers (13.3\% respondents). See Table 6.

Table 6. Respondents Distribution by Establishments

\begin{tabular}{|l|c|c|c|c|c|c|c|}
\hline Selected Hotel & $\begin{array}{c}\text { Frontline } \\
\text { Employees (r1) }\end{array}$ & $\begin{array}{c}\% \text { of } \\
(\mathbf{r} 1)\end{array}$ & $\begin{array}{c}\text { Managers } \\
(\mathrm{r} 2)\end{array}$ & $\begin{array}{c}\% \text { of } \\
(\mathrm{r} 2)\end{array}$ & $\begin{array}{c}\text { Customers } \\
(\mathrm{r} 3)\end{array}$ & $\begin{array}{c}\% \text { of } \\
(3)\end{array}$ & $\begin{array}{c}\Sigma \\
(\mathrm{r})\end{array}$ \\
\hline Hotel A & 9 & 8.6 & 12 & 11.4 & 12 & 11.4 & 31.4 \\
\hline Hotel B & 10 & 9.5 & 11 & 10.5 & 14 & 13.3 & 33.3 \\
\hline Hotel C & 12 & 11.4 & 11 & 10.5 & 14 & 13.3 & 33.3 \\
\hline Total & 31 & 29.5 & 34 & 32.4 & 40 & 38.1 & 100 \\
\hline
\end{tabular}

n=105; Source: Field Survey, 2017.

\section{Analyses of Responses}

Means $(\bar{X})$ and standard deviation (SD) were calculated for data generated from field survey, as presented in Table 7, which is determined by;

$\sum \frac{\sqrt{(X-\bar{X})^{2}}}{n-1}$

Where:

$X=$ each data in the set

$\bar{X}=$ Mean

$n=$ total number of data in the set (Oloyo, 2001). Hence,

$\bar{X}$ is given by:

$\frac{\sum F X}{F}$

while SD is given by:

$\frac{\sqrt{X-\bar{X}}}{n}$ 
Therefore, item 1a in Table 7, for instance, is thus calculated;

\begin{tabular}{lllll}
$\mathrm{F}$ & $\mathrm{X}$ & $\mathrm{FX}$ & $(\mathrm{X}-\bar{X})$ & $(\mathrm{X}-\bar{X})^{2}$ \\
21 & 4 & 84 & 0.45 & 0.20 \\
8 & 3 & 24 & -0.55 & 0.30 \\
$\mathrm{O}$ & 2 & $\mathrm{O}$ & -3.55 & 12.6 \\
2 & 1 & 2 & -2.55 & 6.5 \\
\hline 31 & 4 & 110 & & 19.6 \\
& & & & \\
$\bar{X}=3.55$ & & $S D=\frac{\sqrt{19.6}}{4}$ & & $S D=1.11$
\end{tabular}

Table 7. Analyses of Responses from Questionnaire

\begin{tabular}{|c|c|c|c|c|c|c|}
\hline \multirow{2}{*}{ Statement } & \multicolumn{6}{|c|}{ Responses } \\
\hline & SA (\%) & $A(\%)$ & $\mathrm{D}(\%)$ & SD (\%) & Mean & Std. Deviation \\
\hline \multicolumn{7}{|c|}{ 1. Conflict of Interest Sub-scale $(n=31)$} \\
\hline a. Group Conflict & $21(67.7)$ & $\begin{array}{c}8 \\
(25.8)\end{array}$ & - & $\begin{array}{c}2 \\
(6.5)\end{array}$ & 3.55 & 1.11 \\
\hline b. Incompatible Requests & $20(64.5)$ & $\begin{array}{c}8 \\
(25.8)\end{array}$ & - & $\begin{array}{c}3 \\
(9.7)\end{array}$ & 3.45 & 0.73 \\
\hline c. Resentment Among Employees & $\begin{array}{c}8 \\
(25.8)\end{array}$ & $13(41.9)$ & - & $10(32.3)$ & 2.61 & 0.56 \\
\hline d. Staff-guest Conflict & $12(38.7)$ & $11(35.5)$ & $\begin{array}{c}8 \\
(25.8)\end{array}$ & - & 3.13 & 0.65 \\
\hline e. Workplace Tension & $\begin{array}{c}4 \\
(12.8)\end{array}$ & $20(64.6)$ & $\begin{array}{c}7 \\
(22.6) \\
\end{array}$ & - & 2.91 & 0.6 \\
\hline \multicolumn{7}{|c|}{ 2. Role Conflict and Role Ambiguity Sub-scale $(n=31)$} \\
\hline a. Authority Assurance & $\begin{array}{c}2 \\
(6.4)\end{array}$ & $\begin{array}{c}3 \\
(9.7)\end{array}$ & $15(48.4)$ & $11(35.5)$ & 1.87 & 0.64 \\
\hline b. Unclear Objectives & $\begin{array}{c}6 \\
(19.3)\end{array}$ & $\begin{array}{c}4 \\
(12.9)\end{array}$ & $\begin{array}{c}16 \\
(5.6)\end{array}$ & $5(16.2)$ & 2.35 & 0.56 \\
\hline c. Loss of Ideas & $14(45.1)$ & $17(54.9)$ & - & - & 3.45 & 0.73 \\
\hline d. Inadequate Manpower & $15(48.4)$ & $14(45.2)$ & $\begin{array}{c}2 \\
(6.4)\end{array}$ & - & 3.42 & 0.72 \\
\hline e. Role Clarity & $\begin{array}{c}4 \\
(12.9)\end{array}$ & $\begin{array}{c}1 \\
(3.2)\end{array}$ & $14(45.2)$ & $12(38.7)$ & 1.90 & 0.63 \\
\hline f. Policy Inflexibility & $18(58.1)$ & $\begin{array}{c}4 \\
(12.9)\end{array}$ & $\begin{array}{c}3 \\
(9.7)\end{array}$ & $6(19.3)$ & 3.09 & 0.63 \\
\hline g. Inadequate Resources & $16(51.6)$ & $\begin{array}{c}3 \\
(9.7) \\
\end{array}$ & $12(38.7)$ & - & 3.13 & 0.64 \\
\hline h. Clear-cut Assignment & $\begin{array}{c}3 \\
(9.7) \\
\end{array}$ & $\begin{array}{c}6 \\
(19.3)\end{array}$ & $15(48.4)$ & $7(22.6)$ & 2.16 & 0.59 \\
\hline \multicolumn{7}{|l|}{ 3. Work Scheduling Sub-scale $(n=31)$} \\
\hline a. Unstable Working Hours & $20(64.5)$ & $\begin{array}{c}7 \\
(22.5)\end{array}$ & $\begin{array}{c}2 \\
(6.5)\end{array}$ & $\begin{array}{c}2 \\
(6.5)\end{array}$ & 3.45 & 0.73 \\
\hline b. Excessive Stress & $19(61.3)$ & - & $12(38.7)$ & - & 3.23 & 0.67 \\
\hline
\end{tabular}




\begin{tabular}{|c|c|c|c|c|c|c|}
\hline \multirow{2}{*}{ Statement } & \multicolumn{6}{|c|}{ Responses } \\
\hline & SA (\%) & $A(\%)$ & $\mathrm{D}(\%)$ & SD (\%) & Mean & Std. Deviation \\
\hline c. Weekend Job Strain & $14(45.2)$ & $\begin{array}{c}9 \\
(29.0)\end{array}$ & $\begin{array}{c}8 \\
(25.8)\end{array}$ & - & 3.19 & 0.66 \\
\hline d. Shiftwork and Poor Leisure & $17(54.8)$ & $10(32.3)$ & - & $4(12.9)$ & 3.29 & 0.68 \\
\hline \multicolumn{7}{|l|}{ 4. Pay Satisfaction Sub-scale $(n=31)$} \\
\hline a. Pay and Benefit Packages & $\begin{array}{c}2 \\
(6.5)\end{array}$ & $\begin{array}{c}5 \\
(16.1)\end{array}$ & $24(77.4)$ & - & 1.29 & 0.82 \\
\hline b. Low Pay Replacement & $19(61.3)$ & - & $12(38.7)$ & - & 3.23 & 0.67 \\
\hline c. Pay Dissatisfaction & $19(61.3)$ & - & $12(38.7)$ & - & 3.23 & 0.67 \\
\hline d. Salary Advancement Certainty & - & $\begin{array}{c}8 \\
(25.8)\end{array}$ & $\begin{array}{c}2 \\
(6.5)\end{array}$ & $21(67.7)$ & 1.58 & 0.72 \\
\hline \multicolumn{7}{|l|}{ 5. Work-Family Conflict Sub-scale $(n=31)$} \\
\hline a. Work-Private-Life Conflict & $12(38.7)$ & $14(45.2)$ & $5(16.1)$ & - & 3.23 & 0.67 \\
\hline b. Pressure from Family Responsibility & $22(71.0)$ & - & $\begin{array}{c}9 \\
(29.0)\end{array}$ & - & 3.42 & 0.72 \\
\hline c. Lack of Support to Family Activities & $22(71.0)$ & - & $\begin{array}{c}9 \\
(29.0)\end{array}$ & - & 3.42 & 0.72 \\
\hline d. Perceived Home Stressors & $19(61.3)$ & - & $12(38.7)$ & - & 3.23 & 0.67 \\
\hline e. Zero Job Stressors & - & $12(38.7)$ & $19(61.3)$ & - & 2.39 & 0.56 \\
\hline \multicolumn{7}{|c|}{ 6. Employee Job Commitment Sub-scale $(n=31)$} \\
\hline a. Lack of Employee Job Involvement & $17(54.9)$ & $13(41.9)$ & $\begin{array}{c}1 \\
(3.2) \\
\end{array}$ & & 3.61 & 0.79 \\
\hline b. Negative Career Identity & $11(35.5)$ & $16(51.6)$ & $\begin{array}{c}4 \\
(12.9)\end{array}$ & - & 3.23 & 0.67 \\
\hline c. Job Satisfaction & - & $10(32.3)$ & - & $21(67.7)$ & 1.65 & 0.70 \\
\hline d. Job Displeasure & $21(67.7)$ & - & $10(32.3)$ & - & 3.35 & 0.70 \\
\hline \multicolumn{7}{|c|}{ 7. Service Quality and Customer Satisfaction Sub-scale $(n=40)$} \\
\hline a. Poor Staff-guest Relationship & $18(45.0)$ & $14(35.0)$ & $\begin{array}{c}8 \\
(20.0)\end{array}$ & - & 3.25 & 0.67 \\
\hline b. Poor Overall Customer Satisfaction & $20(50.0)$ & $13(32.5)$ & $\begin{array}{c}5 \\
(12.5) \\
\end{array}$ & $\begin{array}{c}2 \\
(5.0) \\
\end{array}$ & 3.26 & 0.70 \\
\hline c. Charges for Quality Service & $5(12.5)$ & $\begin{array}{c}2 \\
(5.0)\end{array}$ & $10(25.0)$ & $23(57.5)$ & 1.73 & 0.68 \\
\hline d. Employee Friendliness & $5(12.5)$ & $\begin{array}{c}3 \\
(7.5) \\
\end{array}$ & $12(30.0)$ & $20(50.0)$ & 1.83 & 0.65 \\
\hline e. Service Inefficiency & $12(30.0)$ & $16(40.0)$ & $12(30.0)$ & - & 3.0 & 0.61 \\
\hline \multicolumn{7}{|c|}{ 8. Organizational Performance Sub-scale $(n=34)$} \\
\hline a. Occupancy Level Non-improvement & $16(47.06)$ & $9(26.47)$ & $9(26.47)$ & - & 3.21 & 0.66 \\
\hline b. High Revenue From Occupancy & - & $11(32.3)$ & $16(47.1)$ & $7(20.6)$ & 2.12 & 0.59 \\
\hline \multicolumn{7}{|l|}{ 9. Intention to Leave Sub-scale $(n=31)$} \\
\hline a. Turnover Culture & $16(51.6)$ & $10(32.3)$ & $\begin{array}{c}5 \\
(16.1)\end{array}$ & - & 3.35 & 0.70 \\
\hline b. Organizational Loyalty & - & $5(16.1)$ & $10(32.3)$ & $16(51.6)$ & 1.65 & 0.70 \\
\hline c. Action Tendency Measure & $13(41.9)$ & $15(48.4)$ & $\begin{array}{c}3 \\
(9.7)\end{array}$ & - & 3.32 & 0.69 \\
\hline
\end{tabular}




\begin{tabular}{|l|c|c|c|c|c|c|}
\hline \multirow{2}{*}{ Statement } & \multicolumn{5}{|c|}{ Responses } \\
\cline { 2 - 8 } d. Retention Tendency & SA (\%) & A (\%) & D (\%) & SD (\%) & Mean & Std. Deviation \\
\hline 10. Causes of Conflict Sub-scale $(n=34)$ & - & $\begin{array}{c}8 \\
(25.8)\end{array}$ & $\begin{array}{c}7 \\
(22.6)\end{array}$ & $16(51.6)$ & 1.74 & 0.68 \\
\hline a. Pressure from Unclear Responsibilities & $17(50.0)$ & $12(35.3)$ & $\begin{array}{c}5 \\
(14.7)\end{array}$ & - & 3.35 & 0.70 \\
\hline b. Adequate Reward System & - & $\begin{array}{c}5 \\
(14.7)\end{array}$ & $11(32.4)$ & $18(52.9)$ & 1.62 & 0.71 \\
\hline c. Family Influence & $25(73.53)$ & $8(23.53)$ & $\begin{array}{c}1 \\
(2.94)\end{array}$ & - & 3.71 & 0.82 \\
\hline d. Recurrent Customer Complaint & $17(50.0)$ & $\begin{array}{c}12(35.3) \\
(14.7)\end{array}$ & - & 3.35 & 0.70 \\
\hline e. Employee Confrontation & $14(41.18)$ & $15(44.12)$ & $4(11.76)$ & $1(2.94)$ & 3.24 & 0.67 \\
\hline f. Irregularities in work Settings & - & $26(76.47)$ & $8(23.53)$ & - & 2.76 & 0.57 \\
\hline
\end{tabular}

Source: Field Survey, 2017.

Scale Code: $S A=$ Strongly Agreed; $A=$ Agreed; $D=$ Disagreed; $S D=$ Strongly Disagreed .

Decision Rule: Respondents disagree if Mean $\leq 2.49$; Respondents agree if Mean $\geq 2.50$.

Decision is based on effective sample size ( $\leq 100)$ for each sub-scale (Egbulonu, 2001)

\section{Test of Hypotheses}

The Chi-square is calculated as:

$$
x^{2}=\sum_{i=1}^{m} \frac{(O i-E i)^{2}}{E i} \quad \text { Bladock, 1998) }
$$

Level of significance for hypotheses test is at $5 \%$, meaning that the probability of rejection of each hypothesis or if it is true; that is, type 1 error, is fixed at; 0.05 .

Decision: The statistical representation of decision rule is given that if $\mathrm{X}^{2}$ cal $>\mathrm{X}^{2}$ tab, reject Ho. Rejecting Ho means that there is significant relationship between two variables, while accepting it means that there is no significant relationship between two variables.Similar test method was applied in the test of the hypotheses. In testing $\mathrm{H}_{1}$ for instance;

Table 8. Contingency Table for Hypothesis One

\begin{tabular}{|c|c|c|c|}
\hline From Table 9 & SA \& A & D \& SD & Row Total \\
\hline $6 a$ & 30 & 1 & 31 \\
\hline $6 b$ & 27 & 4 & 31 \\
\hline $6 c$ & 10 & 21 & 31 \\
\hline $6 d$ & 21 & 10 & 31 \\
\hline Column Total & 88 & 36 & 124 \\
\hline \multicolumn{2}{|c|}{ Grand Total } & 124 \\
\hline
\end{tabular}

Source: Tables 9 (item 6 a, b,c,d) Field Survey; 2017 
The expected value " $\mathrm{E}$ " is calculated using the compound proportion model, according to Ogoamaka (1998):

$(\mathrm{rt} \cdot \mathrm{ct}) / \mathrm{gt}$

Where: $\mathrm{rt}=$ row total; $\mathrm{ct}=$ column total; $\mathrm{gt}=$ grand total

From Table 7 (6a)

yes: $\mathrm{E}=(31 \cdot 88) / 124=22$

no: $\mathrm{E}=(31 \cdot 36) / 124=9$

From Table 7 (6b)

yes: $\mathrm{E}=(31 \cdot 88) / 124=22$

no: $\mathrm{E}=(31 \cdot 36) / 124=9$

From Table 7 (6c)

yes: $\mathrm{E}=(31 \cdot 88) / 124=22$

no: $\mathrm{E}=(31 \cdot 36) / 124=9$

From Table 7 (6d)

yes: $\mathrm{E}=(31 \cdot 88) / 124=22$

no: $\mathrm{E}=(31 \cdot 36) / 124=9$

Table 9. Statistical Table for Hypothesis One

\begin{tabular}{|c|c|c|c|c|}
\hline $\mathrm{O}$ & $\mathrm{E}$ & $0-\mathrm{E}$ & $(\mathrm{O}-\mathrm{E})^{2}$ & $(\mathrm{O}-\mathrm{E})^{2} / \mathrm{E}$ \\
\hline 31 & 22 & 9 & 81 & 3.7 \\
\hline 1 & 9 & -8 & 64 & 7.1 \\
\hline 27 & 22 & 5 & 25 & 1.14 \\
\hline 4 & 9 & -5 & 25 & 2.8 \\
\hline 21 & 22 & -1 & 1 & 0.05 \\
\hline 10 & 9 & 1 & 1 & 0.11 \\
\hline 10 & 22 & -12 & 144 & 6.5 \\
\hline 21 & 9 & 12 & 144 & 16.1 \\
\hline & \multicolumn{5}{|r}{$X^{2}$ Value } \\
\hline
\end{tabular}

Source: Field Survey, 2017.

Critical Value: $(\mathrm{r}-1) \quad(\mathrm{c}-1)$

$(4-1) \quad(2-1)$

(3) (1)

$\mathrm{df}=3$ 
Table 10. Statistical Table for Hypothesis Two

\begin{tabular}{|c|c|c|c|c|}
\hline $\mathrm{O}$ & $\mathrm{E}$ & $\mathbf{0}-\mathrm{E}$ & $(\mathrm{O}-\mathrm{E})^{2}$ & $(\mathrm{O}-\mathrm{E})^{2} \div \mathrm{E}$ \\
\hline 25 & 18 & 7 & 49 & 2.7 \\
\hline 9 & 16 & -7 & 49 & 3.1 \\
\hline 11 & 18 & -7 & 49 & 2.7 \\
\hline 23 & 16 & 7 & 49 & 3.1 \\
\hline \multicolumn{5}{|c}{$\mathrm{X}^{2}$ Value } \\
\hline
\end{tabular}

Source: Field Survey, 2017.

Table 11. Statistical Table for Hypothesis Three

\begin{tabular}{|c|c|c|c|c|}
\hline$O$ & $E$ & $0-E$ & $(O-E)^{2}$ & $(O-E)^{2} / E$ \\
\hline 32 & 21.6 & 10.4 & 108.16 & 5.01 \\
\hline 8 & 18.4 & -10.4 & 108.16 & 5.9 \\
\hline 33 & 21.6 & 11.4 & 129.96 & 6.02 \\
\hline 7 & 18.4 & -11.4 & 129.96 & 7.1 \\
\hline 7 & 21.6 & -14.6 & 213.16 & 9.9 \\
\hline 33 & 18.4 & 14.6 & 213.16 & 11.6 \\
\hline 8 & 21.6 & -13.6 & 184.96 & 8.6 \\
\hline 32 & 18.4 & 13.6 & 184.96 & 10.1 \\
\hline 28 & 21.6 & 6.4 & 40.96 & 1.9 \\
\hline 12 & 18.4 & -6.4 & 40.96 & 2.23 \\
\hline
\end{tabular}

Source: Field Survey, 2017.

Table 12. Statistical Table for Hypothesis Four

\begin{tabular}{|c|c|c|c|c|}
\hline $\mathrm{O}$ & $\mathrm{E}$ & $0-\mathrm{E}$ & $(\mathrm{O}-\mathrm{E})^{2}$ & $(\mathrm{O}-\mathrm{E})^{2} / \mathrm{E}$ \\
\hline 26 & 16.8 & 9.2 & 84.6 & 5.04 \\
\hline 5 & 14.3 & -9.3 & 86.5 & 6.05 \\
\hline 5 & 16.8 & -11.8 & 139.2 & 8.29 \\
\hline 26 & 14.3 & 11.7 & 136.9 & 9.57 \\
\hline 28 & 16.8 & 11.2 & 125.4 & 7.46 \\
\hline 3 & 14.3 & -11.3 & 127.7 & 8.93 \\
\hline 8 & 16.8 & -8.8 & 77.4 & 4.61 \\
\hline 23 & 14.3 & 8.7 & 75.7 & 5.29 \\
\hline \multicolumn{5}{|c|}{$X^{2}$ Value } \\
\hline
\end{tabular}

Source: Field Survey, 2017. 


\section{Result of findings}

A three-stage research model for causes, behaviours and outcomes of organizational conflict in the hospitality industry was proposed for the study. The first stage elicits the major variables that cause organizational conflict in the hospitality industry (Table 7 , item 1 to 5 ) and the subsequent areas of impact of the conflict variables, which translate to a number of factors; like conflict of interest (interpersonal conflict) as posited by Crossman (2014) and Stern (2014), role conflict and role ambiguity, work scheduling, pay satisfaction, and work-family conflict; causing organizational conflict and creating impact on such dependent variables as, employee job commitment, service quality, customer satisfaction, and organizational performance (Table 7, item 6 to 8).

The second stage continues with the first stage and discovers the behaviours and outcomes of organizational conflict arising from the impact on the organizational conflict variables. Therefore, the model depicts the causes of organizational conflict as; conflict of interest arising from lack of group cohesion, role conflict and role ambiguity, work schedule, pay satisfaction, and work-family conflict. These conflicts causatives give rise to conflict behaviours such as negative employee job satisfaction, and low level of employee job commitment (Table 7, item 6). The behaviours further develop the following outcomes - poor service quality, negative customer satisfaction and poor organizational performance, and finally, employees' intention to leave the job (Table 7 , item 7 to 9). Note the emphasis that conflict should not be shrugged off by a mere "it will pass..." (Barling, Cooper, 2008; Tannen, 2013).

The third stage relates to the employees' intention to leave the job, further creating conflict within the organization (Table 7 , item 9). Since intention to leave is voluntary within the individual, the continuous emotional stress brought about by this trait further creates discomfort within the employee thus causing varieties of conflict in the organization.

Results further reveal that conflict of interest, role conflict and ambiguity, work scheduling, pay satisfaction, and work-family conflict are the major causes of organizational conflict in the hospitality industry (Table 7, item 10). The existence of these conflicts give rise to behaviours that negatively influence employee job satisfaction and job commitment levels thereby producing outcomes that affect service quality, customer satisfaction and overall organizational performance which inevitably leads to employees' intention to leave the job. See Figure 1.

\section{Chi-square Test Results for the Hypotheses:}

$\mathbf{H}_{\mathbf{1}}$ was tested at 0.05 level of significance and critical value of 7.814 from chi-square table with $3 \mathrm{df}$. Result showed that $\mathrm{X}^{2} \mathrm{cal}(37.5)>\mathrm{X}^{2} \operatorname{tab}$ (7.814). The hypothesis was rejected to confirm that there is a significant impact of organizational conflict on employees' job performance in the hospitality industry.

$\mathbf{H}_{2}$ was tested at 0.05 level of significance and critical value of 3.841 from chi-square table with $1 \mathrm{df}$. Result revealed that $\mathrm{X}^{2} \mathrm{cal}(11.6)>\mathrm{X}^{2} \operatorname{tab}$ (3.841). The hypothesis was rejected; hence, there is a significant impact of organizational conflict on the overall performance of the hospitality organization.

$\mathbf{H}_{3}$ was tested at 0.05 level of significance and critical value of 9.847 from chi-square table with 4 df. The result showed that $X^{2}$ cal (68.36) $>X^{2} \operatorname{tab}$ (9.847). The hypothesis was rejected to affirm that behaviours of employee poor commitment have negative effect on service quality and customer satisfaction in the hospitality industry.

$\mathbf{H}_{4}$ was tested at 0.05 level of significance and critical value of 7.814 from chi-square table with $3 \mathrm{df}$. The result showed that $\mathrm{X}^{2} \mathrm{cal}(55.24)>\mathrm{X}^{2} \operatorname{tab}$ (7.814). The hypothesis was rejected in line with the decision rule showing a significant relationship between organizational conflict and employees' intention to leave. 


\section{Conclusion}

This research has clearly shown that significant relationships exist between organizational conflict and employee job performance, customer satisfaction, organizational performance, and employees' intention to leave the current job in the hotel industry. Organizational conflict arises in the hospitality industry from a number of factors including lack of group cohesiveness, employee dissatisfaction arising from unfair pay packages, emotional exhaustion, and other dimensions of role interference and work-family conflict. For the hospitality industry, information from this research can be used to evaluate and establish preventive or corrective actions with respect to role related factors such as work overload. In addition, role conflict and role ambiguity are important factors in increasing the probability that hospitality industry employees will experience conflict and perceive the desirability of leaving the current job.

It was equally revealed that one major impact of organizational conflict on employee job performance clearly manifests in employees' poor commitment towards the job which in turn affect service quality and customer satisfaction. The outcome here is that the performance of the organization is hampered and employee job satisfaction is affected, triggering off turnover intentions.

The hospitality industry is characterized by small working groups who work together to achieve a common goal, depending on the philosophy of the organization. Most of the small groups studied in this research have conflicting interests that put them in persistent misunderstanding with each other while trying to put forth perceived rational ideas towards the job. This is where the managers have failed to enforce team building and cohesiveness among working groups.

Minimal attention has been focused on the role of pay satisfaction, work scheduling, role conflicts, role ambiguity, and work-family conflict as causatives of organizational conflict within the structure of the hospitality industry as well as the outcome of organizational conflict on the job performance of the employee with the resultant effect on employee job satisfaction and commitment, service quality and their role on customer satisfaction, overall organizational performance and employees' intention to leave. This depicts that the impact of organizational conflict on employee job performance in the hospitality industry is significant as it negatively affects overall organizational performance.

\section{Recommendation:}

1. The total number of hours an employee must work should be kept within an acceptable level and should differ since some employees will be able to tolerate more hours than others.

2. Practitioners in the hospitality industry need to re-examine job related tensions and stress which are a direct function of role conflict (direct conflicts in which role obligations must be reconciled) and role ambiguity (lack of role clarity).

3. A kind of reward system should be introduced for employees who work in a group, with benefits that make the group attractive to be in; or they may encourage them to stay in the team by some other means suitable for the organization. The key factor may be competition from other groups within the establishment. The need to achieve set objective for a particular group often enhances group cohesion radically because members have an incentive to stay in the group and to work together to achieve their common goal.

4. Human resource departments (HRD) of hospitality organizations should include well-designed and implemented human resource training programmes for supervisors and managers towards seeking a specific employee retention goal for the organization. 
The HRD should attempt to establish and strengthen the supportive mechanism within the organization since it can reduce the impact of stressors on psychological states and intention to quit.

5. Hospitality organizations might need to implement family-friendly policies that, possibly, will reduce the tension that create work-family stressors for employees. What is more important is that middle managers and line supervisors should be involved in the change effort and be able to communicate policies effectively in a holistic manner.

6. An employee complain consortium should be set up by managements of hospitality outfits to reduce build-up of employee face-offs and minimize reactions arising from unresolved misunderstandings among colleagues. They should design a mechanism to let employees vent frustrations, and post views and opinions. It should be both therapeutic and censorship free; hence any subject may be complained of.

7. Recruitment and selection processes should be designed in a way that individuals who have passion for the hospitality industry are employed for jobs in the industry, rather than recruiting transient employees who exit the industry at the slightest provocation and role misinterpretation.

8. A conflict management unit/team with strong managerial competencies should be put in place in hospitality organizations (within the human resource department) as a proactive approach to forestall incidents of conflicts likely to arise in the workplace. The team may, for instance, assist an employee to manage emotional crisis or in forecasting non-cohesiveness and distrust among working groups capable of generating dimensions of role interference within individuals in the group.

9. Managers of hospitality organizations should be constantly trained on organizational conflict management procedures so that when traces of conflict arise in the organization, managers would be able to manage the situation between employees, and, or between the organization and concerned employee(s) before things get out of hand.

\section{References}

Allen, W.R., Drevs, R.A., Ruhe, J.A. 2009. Reasons why college educated women change employment. Journal of Business and Psychology 14 (1), 77-93.

Almer, E.D., \& Kaplan, S.E. 20oo. Myths and realities of flexible work arrangements. The CPA Journal 70 (4), 14-17, 73-74.

Barling, J., Cooper, C. 2008. The SAGE Handbook of Organizational Behaviour. Los Angeles; SAGE Publications Ltd.

Baroudi, J.J. 2005. The impact of role variables on IS personnel work attitudes and intentions. MIS Quarterly 9 (4), 341-356.

Bedeian, A.G., Armenakis, A.A. 2001. A path analytic study of the consequences of role conflict and ambiguity. Academy of Management Journal 24, 417-424.

Bedeian, A.G., Burke, B.G., \& Moffet, R.G. 2008. Outcomes of work-family conflict among married male and female professionals. Journal of Management 14 (3), 475-491.

Behrman, D.N., Perreault, W.D.J. 2004. A role stress model of the performance and satisfaction of industrial salesperson. Journal of Marketing 14 (3), 475-491.

Berta, D. 2004. Confab Forecast: Labour shortage, turnover to worsen soon. Nation's Restaurant News 38 (8), 16-17 
Birdir, K. 2002. General manager turnover and root causes. International Journal of Contemporary Hospitality Management 14 (1), 43-47.

Bladock, H.M. 1998. Social statistics. New York: McGraw Hill International Book Coy.

Boles, J.S., Babin, B.J. 2006. On the front lines: Stress, conflict, and the customer service provider. Journal of Business Research 37 (1), 41-50.

Boyar, S.L., Maertz, C.P Jr., Pearson, A.W., Keough, S. 2003. Work-family conflict: A model of linkages between work and family domain variables and turnover intentions. Journal of Managerial Issues 15 (2), 175-19o.

Cotton, J.L., Tuttle, J.M. 1986. Employee turnover: A meta-analysis and review with implications for research. Academy of Management Review 11 (1), 57-70.

Crossman, A. 2014. Role conflict. Retrieved from http://sociology.about.com/od/R_Index/g/ Role-Conflict.htm

Deutsch, M., Coleman, P.T., Marcus, E.C. 2011. The handbook of conflict resolution: Theory and practice. New Jersey; John Wiley \& Sons.

Dubinsky, A.J., Hartley, S.W. 2006. Antecedents of retail salesperson performance: A path-analytic perspective. Journal of Business Research 14, 253-268.

Egbulonu, K.G. 2001. Topics in advanced business statistics: A simplified approach. Owerri, Nigeria: Peace Publishers.

Fishbein, M., Ajzen, I. 1985. Belief, attitudes, intention and behaviour: An introduction to theory and research. Reading, MA: Addison-Wesley.

Good, L.K., Page, T.J., Young, C.E. 1996. Assessing hierarchical differences in job-related attitudes and turnover among retail managers. Journal of the Academy of Marketing Science 24 (2), 148-156.

Good, L.K., Sisler, G.F., Gentry, J.W. 1998. Antecedents of turnover intentions among retail management personnel. Journal of Retailing 64 (3), 295-314.

Guthrie, J.P. 200o. Alternative pay practices and employee turnover: An organization economics perspective. Group and Organization Management 25 (4), 419-439.

Hecht, L.M. 2011. Role conflict and role overload: Different concepts, different consequences. Sociological Inquiry 71, 111-121.

Hinkin, T.R., Tracey, J.B. 20oo. The cost of turnover. Cornell Hotel and Restaurant Administration Quarterly 41 (3), 14-21.

Hood, J.C., Milazzo, N. 2013. Shiftwork, stress and wellbeing: How workers and their families cope with shiftwork. Personnel Administrator 29 (December), 95-105.

Howard, W.G., Donofrio, H.H., Boles, J.C. 2004. Inter-domain in work-family, family-work conflict and police work satisfaction. Policing, 27 (3), 380-395.

Jamal, M. 2001. Shift work related to job attitudes, social participation and withdrawal behaviours: A study of nurses and industrial workers. Personnel Psychology 34, 535-547.

Kahn, R., Wolfe, D., Quinn, R., Snoek, J., Rosenthal, R. 2004. Organizational stress: Studies on role conflict and ambiguity. New York: Wiley.

Kazerooni, S.H. 2005. Factors influencing Sizzler management turnover. Unpublished Doctoral, United State International University California.

Lambert, E.G., Hogan, N.L., Barton, S.M. 2001. The impact of job satisfaction on turnover intent: A tool of a structural measurement model using a national sample of workers. Social Science Journal 38 (2), 233-250

Leonard, B. 2001. Majority of workers are dissatisfied with pay. HR Magazine, 35 (6), 35-37.

Linden, R. 2005. Attrition among male and female members of the Royal Canadian Mounted Police. Canadian Police College Journal 9 (1), 86-97. 
Lum, L., Kervin, J., Clark, K., Reid, F., Sirola, W. 2008. Explaining nursing turnover intent: job satisfaction, pay satisfaction, or organizational commitment? Journal of Organizational Behavior 19(3), 305-320.

Maertz, C.P.J., Griffeth, R.W. 2004. Eight motivational forces and voluntary turnover: A theoretical synthesis with implications for research. Journal of Management 30 (5), 667-683.

Maqsood, A., Muhammad, M., Saleem, P. 2012. The impact of work-family conflict and pay on employee job satisfaction with moderating effect of perceived supervision support in Pakistan Hotel Sector. Global Journal of Management and Business Research 12 (6), 1-9

Mitchell, T.R., Holtom, B.C., Lee T.W., Sablynski, C.J., Ere, M. 2001. Why people stay: Using job embeddedness to predict voluntary turnover. Academy of Management Journal 44 (6), 1102-1121.

Netemeyer, R.G., Johnston, M.W., Burton, S. 2000. Analysis of role conflict and role ambiguity in a structural equations framework. Journal of Applied Psychology 65, 148-157.

Nicolaides, A. 2010. Conflict management in hotels. International Research Symposium in Service Management, 3, 1-17. Retrieved form Vaal University database.

Nwokorie, E.C 2016. Service recovery strategies and customer loyalty in selected hotels in Lagos State, Nigeria. Net Journal of Business Management 4 (1) 1- 8.

Nwokorie, E.C., Ezeibe, N. 2016. Service failure as a causative of customer aggression on hospitality industry employees. Basic Research Journal of Business Management and Accounts $5(2), 12-18$.

Ogoamaka, P.M.C. 1998. Descriptive statistics for research students. Ibadan; Wisdom Publishers Limited.

Oloyo, R.A. 2001. Fundamentals of research methodology for social and applied sciences. Ilaro Nigeria; ROA Educational Press.

Orpen, C. 2001. Effect of flexible working hours on employee satisfaction and performance: A field experiment. Journal of Applied Psychology 66 (1), 113-115.

Richer, S., Blanchard, C., Vallerand, R. (2002). A motivational model for turnover. Journal of Applied Psychology, 32 (10) 2089-2113.

Rizzo, J. R., House, R. J., Lirtzman, S. I. 1970. Role conflict and ambiguity in complex organizations. Administrative Science Quarterly 15 (2), 150-163.

Schulz, R.M., Bigoness, W.J., Gagnon, J.P. 2007. Determinants of turnover intentions among retail pharmacists. Journal of Retailing 63 (1), 89-98.

Schawnms, R.C. 1994. Advanced statistics: An introduction. London: Knightbridge Publications Ltd.

Scott, C.R., Connaughton, S.L., Diaz-Saenz, H.R., Maguire, K., Ramirez, R., Richardson, R. (1999). The impact of communication and identifications on intention to leave: A multi-methodological exploration. Management Communication Quarterly 12 (3), 400-435.

Simons, T., Hinkin, T. 2001. The effect of employee turnover on hotel profits: A test across multiple hotels. The Cornell Hotel and Restaurant Administration Quarterly 42 (4), 65-69.

Spector, P.E. 1997. Job satisfaction: Application, assessment, causes, and consequences. Thousand Oaks, CA: SAGE Publications.

Spencer, D.G., Steers, R.M., Mowday, R.T. 2003. An empirical test of the inclusion of job search linkages into Mobley's model of the turnover decision process. Journal of Occupational Psychology 56, 137-144.

Stern, D. 2014. Types of conflict. Retrieved from LIVESTRONG database: www.livestrong.com Stryker, S., Macke, A.S. 2008. Status inconsistency and role conflict. Annual Review of Sociology 4, 57-90. 
Tannen, D. 2013. What is conflict? Definition and assumption about conflict. Retrieved from Academic Leadership Support website: www.ohrd.wisc.edu/onlinetraining/resolution/ aboutwhatisit.htm

Tett, R.P., Meyer, J.P. 1993. Job satisfaction, organizational commitment, turnover intention, and turnover; Path analyses based on meta-analytic findings. Personnel Psychology 46, 259293

Woods, R.H., Heck, W., Sciarini, M. 2008. Turnover, and diversity in the lodging industry. Washington D.C; American Hotel Foundation.

Woods, R.H., Macaulay, J.F. 2009. Rx for turnover: Retention programs that work. The Cornell Hotel and Restaurant Administration Quarterly 30 (1), 78-90. 